\section{Point Dose Measurement for Verification of Treatment Planning System using an Indigenous Heterogeneous Pelvis Phantom for Clarkson, Convolution, Superposition, and Fast Superposition Algorithms}

\author{
Singh S. ${ }^{1 \oplus}$, Raina P. ${ }^{2 *}$, Gurjar O. P. ${ }^{3}$
}

\begin{abstract}
Background: Nowadays, advanced radiotherapy equipment includes algorithms to calculate dose. The verification of the calculated doses is important to achieve accurate results. Mostly homogeneous dosimetric phantoms are available commercially which do not mimic the actual patient anatomy; therefore, an indigenous heterogeneous pelvic phantom mimicking actual human pelvic region has been used to verify the doses calculated by different algorithms.
\end{abstract}

Objective: This study aims to compare the planed dose using different algorithms with measured dose using an indigenous heterogeneous pelvic phantom.

Material and Methods: In this experimental study, various three dimensional conformal radiotherapy (3D-CRT) plans were made using different doses calculated by algorithms. The plans were delivered by medical linear accelerator and doses were measured by ion chamber placed in the indigenous pelvic phantom. Planned and measured doses were compared with together and analyzed.

Results: The relative electron densities of different parts in the pelvic phantom were found to be in good agreement with that of actual pelvic parts, including bladder, rectum, fats and bones. The highest percentage deviations between planned and measured dose were calculated in the single field for Superposition algorithm $(3.09 \%)$ and single field with $45^{\circ}$ wedge for Superposition (3.04\%). The least percentage deviation was calculated in the opposite field for Convolution which was $-0.08 \%$. The results were within the range of $\pm 5 \%$ as recommended by International Commission on Radiation Units and Measurement.

Conclusion: The cost-effective indigenous heterogeneous pelvic phantom has the density pattern similar to the actual pelvic region; thus, it can be used for routine patient-specific quality assurance.

Citation: Singh S, Raina P, Gurjar O. P. Point Dose Measurement for Verification of Treatment Planning System using an Indigenous Heterogeneous Pelvis Phantom for Clarkson, Convolution, Superposition, and Fast Superposition Algorithms. J Biomed Phys Eng. 2019;9(6):613-620. https://doi.org/10.31661/jbpe.v0i0.1185.

\section{Keywords}

Algorithms; Dosimetric Phantoms; Radiotherapy, Conformal; Particle Accelerators, Pelvis

\section{Introduction}

he success of the radiotherapy depends on the accurate delivery of radiation dose to the target volume. Therefore, it is crucial to ensure that the specified target dose is delivered as accurately
${ }^{1} \mathrm{PhD}$, Department of Physics, Ranchi University, Ranchi- 834008, Jharkhand, India

${ }^{2}$ DipRP, Research Schol-

ars, University Depart-

ment of Physics, Ranchi

University, Ranchi-

834008, Jharkhand

State, India

${ }^{3} \mathrm{PhD}$, Department of

Radiotherapy, AlIMS,

Bhopal- 462020, Mad-

hya Pradesh, India

*Corresponding author:

P. Raina

Research Scholars,

University Department of

Physics, Ranchi Univer-

sity, Ranchi- 834008,

Jharkhand State, India

E-mail: payalrai-

na2008@gmail.com

Received: 18 May 2019

Accepted: 27 June 2019 
as possible [1] There are many Quality Assurance (QA) protocols ensuring that all treatment facilities used in radiotherapy are properly checked for accuracy and consistency [2]. Treatment Planning System (TPS) plays a key role in radiotherapy. It ensures that patients receive the prescribed doses precisely for which a number of correction factors are applied [3]. The functionality and quality of TPS are dependent on the type of algorithms used in the different steps of the planning process $[4,5]$. There are several algorithms for TPS playing the central role of calculating dose at any position inside the patient. The algorithm is defined as a sequence of instructions operating on a set of input data, transforming information into a set of output results that are of interest to the user [5-7]. Many algorithms are used in the treatment planning process. The most well-known algorithm is the dose calculation algorithm calculating the dose at any point within the patient in consideration of the patient and beam characteristics [8]. It is of paramount importance for the modern conformal radiotherapy technique to have accuracy in dose calculations in almost all relevant clinical situations. Therefore, a scheduled quality assurance program should be established to verify the plans made on TPS.

The study aims to compare the planed dose with measured dose from four different algorithms, using Three-Dimensional Conformal Radiotherapy Technique (3D-CRT). In this study Clarkson, Convolution, Superposition, and Fast superposition algorithms were applied for all plans to verify whether our indigenous heterogeneous pelvic phantom is suitable for routine quality assurance (QA). This allowed us to know the suitability of an algorithm for the respective diagnostic and treatment technique. This indigenous heterogeneous pelvic phantom is used for the study because the cost of commercial pelvic phantom causes a burden in small radiotherapy centers for quality assurance. The materials used for the construction of heterogeneous phantom are not expensive.

\section{Material and Methods}

Phantom design

In this experimental study, an indigenous heterogeneous phantom was designed in the shape of the human pelvic region. This phantom was constructed by wax, pelvic bone, borax powder and water. To construct the phantom, first pelvic bone (equivalent to human bone) was placed in a cylindrical shaped container. After placing it, a round plastic container filled with water was placed for bladder. Borax powder, glue and water for rectum were placed below the bladder. Wax was poured into it and allowed to solidify. After complete solidification of the wax, the outer container was cut and removed. A cavity was prepared at approximately geometrical center of phantom for $0.6 \mathrm{cc}$ Farmer type Chamber (PTWFreiburg TN30013) and kept at the same position till the end of experiment. Three fiducially lead markers were put on two bilateral points, one anterior and one on surface of phantom. The relative electron density was measured in Monaco treatment Planning version 3.1 (Elekta Ltd, Crawley, UK) for artificial pelvic bone, wax for fat, air, water, borax powder [9]. Volume of interest (VOI) diameter was $1 \mathrm{~cm}$ and volume $0.524 \mathrm{~cm}^{3}$ have been taken for the measurement.

\section{Techniques}

CT scan of heterogeneous phantom was taken and the image was transferred to the Monaco system version 3.1 (Elekta Ltd, Crawley, UK) for contouring. Ion chamber and phantom body were contoured. After contouring, CT images were transferred to the $\mathrm{XiO}$ version 5.10.00.4 (Elekta Ltd, Crawley, UK) planning system. Several simple plans of single to multiple beams were made on the phantom using the different calculation algorithms configured to give 1.0 Gray (Gy) at the isocenter with a $10 \mathrm{~cm} \times 10 \mathrm{~cm}$ field size. Beam isocenter was placed at the centre of effective area of ion chamber. Plans were then transferred to the 
pre-calibrated linear accelerator for measurements.

\section{Dose Calculation Algorithms \\ Clarkson Algorithm}

Clarkson algorithm is a measurement-based method using measured data e.g. PDD and beam profile obtained in a cube water phantom and various corrections are applied to calculate dose distributions [10].

\section{Convolution algorithm}

The convolution algorithm considers the transport of primary photons and that of the scatter photon and electron emerging from the primary photon interaction. The distribution of the dose is calculated from the total energy released per unit mass (TERMA) [10]. The dose $D(\vec{r})$ at a point $\vec{r}$ is given by [11-12].

$$
D(\vec{r})=\int \frac{\mu}{\rho} \psi_{\rho}\left(\overrightarrow{r^{\prime}}\right) A\left(\vec{r}-\left(\overrightarrow{r^{\prime}}\right)\right) d^{3}(\vec{r})
$$

Where, $\frac{\mu}{\rho}$ is mass attenuation coefficient, $\psi_{\rho}$ is primary photon energy fluence, $A\left(\vec{r}-\left(\vec{r}^{\prime}\right)\right)$ is Convolution kernel, the distribution of fraction energy imparted per unit volume.

\section{Superposition algorithm}

The XiO superposition dose deposition method is an adaptation of the "collapsed cone" dose calculation method [13]. Unlike the FFT convolution algorithms, the superposition algorithm energy deposition kernels can be modified to account for variations in electron density [12-13].

\section{Fast superposition algorithm}

Spherical kernels, or "dose spread arrays", are cylindrically symmetric and defined in terms of rays traced along zenith and azimuth angles. The spherical kernel computation has been augmented with the ability to combine (select and sum) adjacent zenith rays in the kernel [14].
Dose reporting and evaluation

Plans were then transferred to the ELEKTA Synergy linear accelerator for measurements. Measurements were carried out with 6 MV photon beam as planned. A pre calibrated Farmer type $0.6 \mathrm{~cm}^{3}$ ionization chamber TN30013 (PTW Dosimetry, Freiburg) along with its electrometer UNIDOSE were used to measure the absorbed dose delivered. Three measurements were made for each plan using the different algorithms for comparison and limitation of statistical uncertainties. Absorbed dose at reference depth was calculated according to the Technical Reports Series No. 398 (TRS 398) of the International Atomic Energy Agency (IAEA) as follows [15].

$\mathrm{D}=\mathrm{M}_{\mathrm{Q}} \times \mathrm{N}_{\mathrm{D}, \mathrm{W}} \times \mathrm{K}_{\mathrm{Q}, \mathrm{Qo}} \times \mathrm{K}_{\mathrm{T}, \mathrm{P}} \times \mathrm{K}_{\mathrm{S}} \times \mathrm{K}_{\mathrm{pol}}$

Where, $M_{Q}$ was the electrometer reading (charge). $\mathrm{N}_{\mathrm{D}, \mathrm{W}}$ and $\mathrm{K}_{\mathrm{Q}, \mathrm{Qo}}$ were the chamber calibration factor and chamber specific factor, respectively. In addition, $\mathrm{K}_{\mathrm{T}, \mathrm{P}}, \mathrm{K}_{\mathrm{S}}$ and $\mathrm{K}_{\mathrm{pol}}$ were temperature pressure correction factor, ion recombination factor and polarization factor, respectively.

Deviation between planned and measured dose was obtained using the equation [16]

$\%$ Deviation $=\frac{D_{m}-D_{\text {ref }}}{D_{\text {ref }}} \times 100$

Where $\mathrm{D}_{\text {ref }}$ and $\mathrm{D}_{\mathrm{m}}$ are calculated dose by the TPS and measured dose result from the designed pelvic phantom, respectively, for this study.

\section{Results}

The relative electron density calculated by the HU number obtained from CT images of pelvic phantom using the TPS lookup table and the relative electron densities of similar organs in actual human pelvic region were given in the Table 1. The relative electron densities for rectum, bladder, air cavity, pelvic bone and fat were $1.068,1.044,0.192,1.455$ and 0.956 , respectively in designed pelvic phantom.

The dose calculated by different algorithms 
Table 1: The density of different organs in indigenous pelvic phantom and the actual patient's pelvis

\begin{tabular}{ccc}
\multirow{2}{*}{ Organs } & \multicolumn{2}{c}{$\begin{array}{c}\text { Relative Electron Density } \pm \\
\text { Standard Deviation }\end{array}$} \\
\cline { 2 - 3 } & Pelvic phantom & Actual human \\
\hline Rectum & $1.068 \pm 0.005$ & $1.067 \pm 0.015$ \\
\hline Bladder & $1.044 \pm 0.004$ & $1.043 \pm 0.008$ \\
\hline Air Cavity & $0.192 \pm 0.196$ & $0.204 \pm 0.221$ \\
\hline Pelvic bone & $1.455 \pm 0.112$ & $1.497 \pm 0.134$ \\
\hline Fat & $0.956 \pm 0.011$ & $0.954 \pm 0.007$
\end{tabular}

on TPS and the measured dose on linear accelerator using pelvic phantom and the ion chamber have been analyzed and given in the Table 2.

\section{Discussion}

To obtain good results of the radiotherapy treatment, it is highly important to deliver the tumoricidal dose to target as planned in the TPS. To verify planned and delivered dose, the dosimetric equipment plays a key role. Most commonly used phantoms for point dose verification are of homogeneous density across their volume which doesn't mimic the density pattern of actual human anatomy [16]. There are few heterogeneous phantoms available commercially that they are costly and available at only few centers in India. Therefore, cost-effective heterogeneous body phantom mimicking the actual human body, by its internal density pattern as well as design, has been highly needed. The indigenously developed phantom used in this study is affordable and the relative electron densities of all the organs designed, as given in Table 1, were approximate-

Table 2: In 3DCRT plans Percentage of variation between planned dose on treatment planning system and measured dose on linear accelerator using pelvic phantom.

\begin{tabular}{|c|c|c|c|c|c|}
\hline & & Clarkson & Convolution & Superposition & Fast Superposition \\
\hline \multirow{3}{*}{ Single field } & $\begin{array}{c}\text { Measured Mean } \\
\text { Dose (cGy) }\end{array}$ & 100.66 & 100.72 & 103.4 & 102.98 \\
\hline & TPS Dose (cGy) & 100.10 & 100.9 & 100.3 & 100.9 \\
\hline & $\%$ Variation & 0.56 & -0.18 & 3.09 & 2.06 \\
\hline \multirow{3}{*}{$\begin{array}{l}\text { Single field with } \\
45 \text { degree wedge }\end{array}$} & $\begin{array}{c}\text { Measured Mean } \\
\text { Dose (cGy) }\end{array}$ & 100.14 & 99.8 & 103.15 & 102.8 \\
\hline & TPS Dose (cGy) & 100.7 & 100.4 & 100.1 & 100.9 \\
\hline & $\%$ Variation & -0.56 & -0.59 & 3.04 & 1.88 \\
\hline \multirow{3}{*}{ Opposite field } & $\begin{array}{c}\text { Measured Mean } \\
\text { Dose (cGy) }\end{array}$ & 100.56 & 100.82 & 103.01 & 102.98 \\
\hline & TPS Dose (cGy) & 100.10 & 100.90 & 100.01 & 100.91 \\
\hline & $\%$ Variation & 0.46 & -0.08 & 2.99 & 2.05 \\
\hline \multirow{3}{*}{ Four field } & $\begin{array}{c}\text { Measured Mean } \\
\text { Dose (cGy) }\end{array}$ & 100.82 & 100.02 & 103.08 & 102.17 \\
\hline & TPS Dose (cGy) & 100.05 & 99.9 & 100.45 & 100.30 \\
\hline & $\%$ Variation & 0.77 & 0.12 & 2.62 & 1.86 \\
\hline
\end{tabular}


ly equal to the corresponding organs of actual human pelvis. The relative electron density of bladder, rectum, fats, bone, and cavities were also estimated by Deepak Shrotriya et al. [17] which were reported as 1.305, 1.0247, 0.9132, 1.5786 and 0.7791 , respectively. These results are also in good agreement with our observed values $1.044,1.068,0.956,1.455$ and 0.192 , respectively with small deviation. Therefore the pelvic phantom designed can be used for the dose verification in the plans done for pelvic region.

The percentage of variations between planned and measured dose were calculated for different field plans as given in Table 2. The percentage of deviations for single field for Clakson(C), Convoluation (Con), Superposition(S) and Fast superposition (FSS) were found to be $0.56 \%,-0.18 \%, 3.09 \%$ and $2.06 \%$, respectively. Percentage of deviations for single field with 45 degree wedge were found to be $-0.56 \%,-0.59 \%, 3.04 \%$ and $1.88 \%$ for $\mathrm{C}$, Con, $\mathrm{S}$ and FSS, respectively. They were, for opposite field for C, Con, S and FSS, $0.46 \%$, $-0.08 \%, 1.99 \%$ and $2.05 \%$, respectively. When four fields were applied for C, Con, S and FSS, percentage of deviations were $0.77 \%, 0.12 \%, 2.62 \%$ and $1.86 \%$, respectively. The highest percentage of deviation was obtained in the single field for superposition algorithm (3.04\%) and wedge field for S (3.04\%), respectively. The least percentage of deviation was obtained in the opposite field for Convolution which was $0.08 \%$ as given in Table 2.

The result for this study using C, FSS and $\mathrm{S}$ algorithms were within $\pm 4 \%$ limit. Results of this study are in consistent with the results published by Muralidhar et al. [14] whose deviations were also within $\pm 4 \%$ limit. The acceptable results must be within the range of $\pm 5 \%$ as recommended by International Commission on Radiation Units and Measurement [18]. A large deviation was obtained using superposition algorithm with single field and wedge field. Since the algorithm could com- pute scattered radiation contribution from the inhomogeneous material. The above result was relatively lower than that obtained by Michael et al. [19]. Dose variation between planned and measured dose, with different field plans using four different algorithms, were shown in Figures 1A to 1D. The dose distribution for superposition algorithm with single field, wedge field, opposite field and four fields were shown in Figures 2A to 2D.

There are some limitations for every algorithm, including density of the material, interactions and dose deposition points, photon and electron contamination, the spectrum assumed to be independent of the field size and shape, the mass attenuation coefficient used in patient, electron contamination assumed to be independent of source to surface distance (SSD), and wedge/block trays in the fluence calculations. Owing to these limitations, it is important to check the accuracy of these algorithms independently. This study evaluates the precision of monitor units obtained by the algorithms used in CMS XiO TPS for heterogeneous pelvic phantom designed to have the routine QA.

\section{Conclusion}

The density pattern of pelvic phantom was in agreement with that of the actual human pelvis; therefore, the materials used in the design of the heterogeneous pelvic phantom were suitable and the phantom can be used for dose verification. Furthermore, designing the phantom was not expensive, and it is easier to use the thimble chambers usually available at each radiotherapy center. Especially smaller radiotherapy centers, which have less income, can locally design such phantom for the dose verification. Making this phantom for dose verification is very useful.

The difference between planned and the measured dose using the heterogeneous pelvic phantom is within the acceptable limit. Therefore, it can be concluded that all the four algorithms calculate dose with the acceptable 

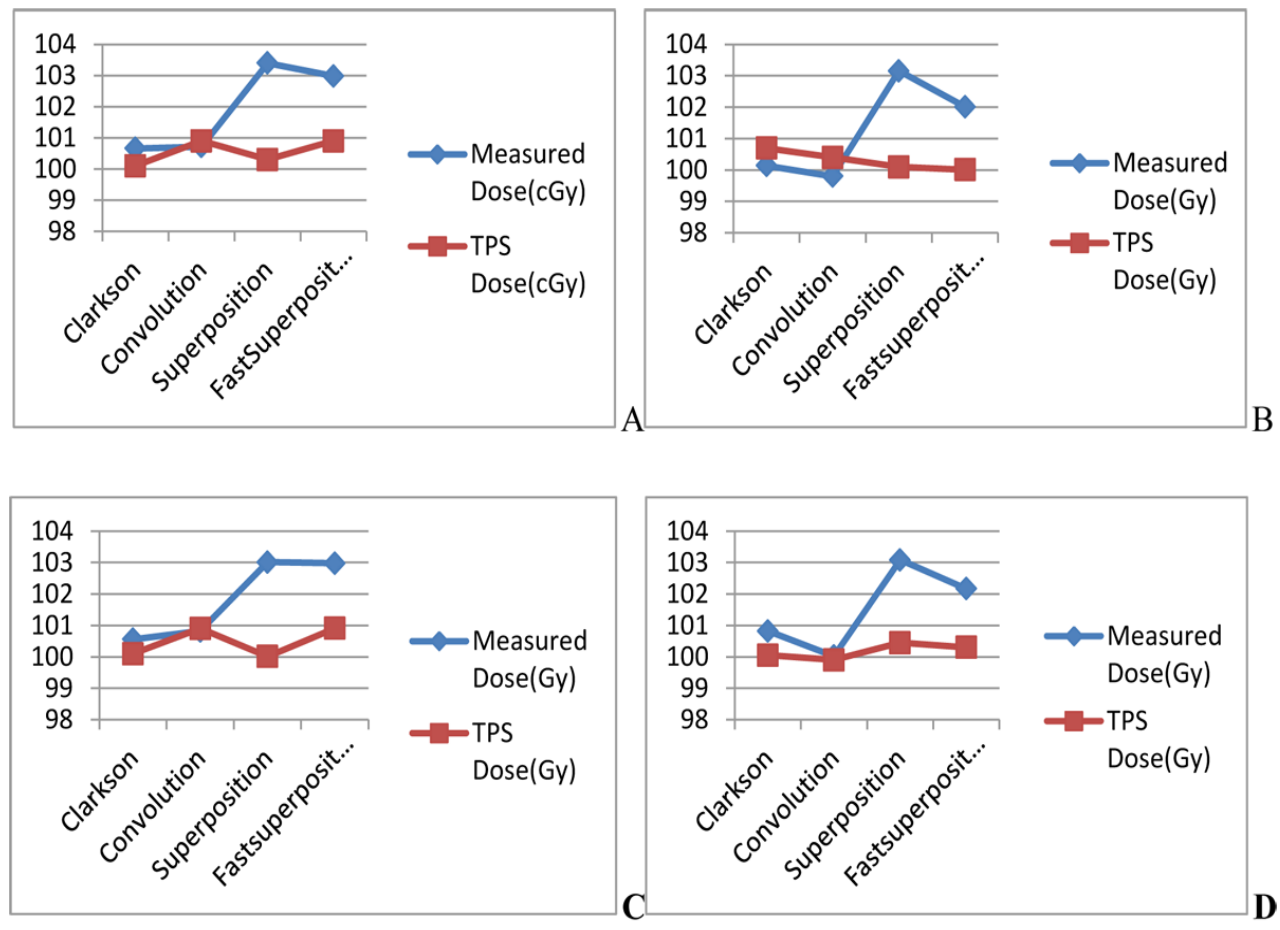

Figure 1: Dose variation between planned dose and measured dose with $10 \times 10 \mathrm{~cm}^{2}$ field size for (A) Single Field B. single field with $45^{\circ}$ Wedge, (C) Opposite field and (D) Four field.
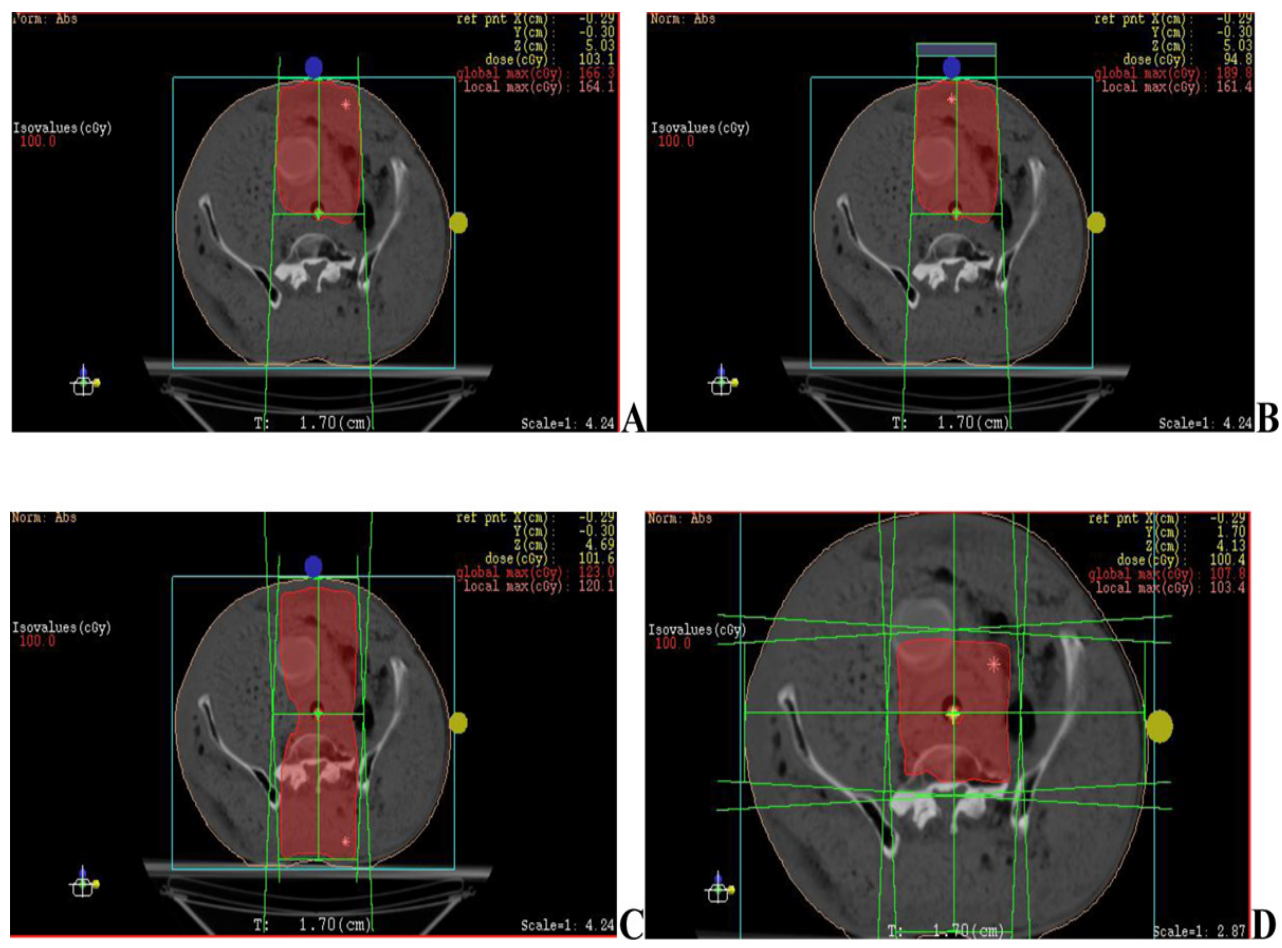

Figure 2: Doses wash with Superposition Algorithm planed with (A) Single Field (B) Wedge Field(C), Opposite Field and (D) Four Field. 
accuracy.

\section{Acknowledgment}

The authors wish to thank the team of radiotherapy department for their thoughtful suggestions.

\section{Conflict of Interest}

None

\section{References}

1. Brahme A. Dosimetric precision requirements in radiation therapy. Acta Radiol Oncol. 1984;23:379-91. doi: 10.3109/02841868409136037. PubMed PMID: 6095609.

2. Mijnheer BJ, Battermann JJ, Wambersie A. What degree of accuracy is required and can be achieved in photon and neutron therapy? Radiother Oncol. 1987;8:237-52. doi: 10.1016/ s0167-8140(87)80247-5. PubMed PMID: 3107087.

3. Nette P, Svensson H. Radiation dosimetry in health care: expanding the reach of global networks. IAEA BULLETIN. 1994;36:33-36.

4. Podgorsak EB. . Radiation oncology physics: $A$ handbook for teachers and students. Vienna: International Atomic Energy Agency; 2005.

5. Van Dyk J, Barnett RB, Cygler JE, Shragge PC. Commissioning and quality assurance of treatment planning computers. Int J Radiat Oncol Biol Phys. 1993;26:261-73. doi: 10.1016/03603016(93)90206-b. PubMed PMID: 8491684.

6. Fraass BA. Quality assurance for 3-D treatment planning. Teletherapy: Present and Future. 1996:253-318.

7. Fraass B, Doppke K, Hunt M, Kutcher G, Starkschall G, Stern R, et al. American Association of Physicists in Medicine Radiation Therapy Committee Task Group 53: quality assurance for clinical radiotherapy treatment planning. Med Phys. 1998;25:1773-829. doi: 10.1118/1.598373. PubMed PMID: 9800687.

8. Brahme A. Design principles and clinical possibilities with a new generation of radiation therapy equipment. A review. Acta Oncol. 1987;26:40312. doi: $10.3109 / 02841868709113708$. PubMed PMID: 3328620.

9. Seco J, Evans PM. Assessing the effect of electron density in photon dose calculations. Med Phys. 2006;33:540-52. doi: 10.1118/1.2161407. PubMed PMID: 16532961.

10.Animesh. Advantages of multiple algorithm support in treatment planning system for external beam dose calculations. J Cancer Res Ther. 2005;1:12-20. PubMed PMID: 17998620.

11. Mackie TR, Scrimger JW, Battista JJ. A convolution method of calculating dose for 15MV x rays. Med Phys. 1985;12:188-96. doi: 10.1118/1.595774. PubMed PMID: 4000075.

12. Boyer AL, Zhu YP, Wang L, Francois P. Fast Fourier transform convolution calculations of $\mathrm{x}$-ray isodose distributions in homogeneous media. Med Phys. 1989;16:248-53. doi: 10.1118/1.596375. PubMed PMID: 2497314.

13. Miften MM, Beavis AW, Marks LB. Influence of dose calculation model on treatment plan evaluation in conformal radiotherapy: a threecase study. Med Dosim. 2002;27:51-7. doi: 10.1016/s0958-3947(02)00088-2. PubMed PMID: 12019966.

14. Muralidhar KR, Murthy NP, Raju AK, Sresty N. Comparative study of convolution, superposition, and fast superposition algorithms in conventional radiotherapy, three-dimensional conformal radiotherapy, and intensity modulated radiotherapy techniques for various sites, done on CMS XIO planning system. J Med Phys. 2009;34:12-22. doi: 10.4103/09716203.48716. PubMed PMID: 20126561; PubMed PMCID: PMCPMC2804143.

15. International Atomic Energy Agency. Absorbed dose determination in external beam radiotherapy: An International Code of Practice for Dosimetry based on standards of absorbed dose to water. Technical Reports Series No. 398. Vienna: International Atomic Energy Agency; 2000. p. 1011-4289.

16. Gurjar OP, Mishra SP, Bhandari V, Pathak P, Patel P, Shrivastav G. Radiation dose verification using real tissue phantom in modern radiotherapy techniques. J Med Phys. 2014;39:449. doi: 10.4103/0971-6203.125504. PubMed PMID: 24600172; PubMed PMCID: PMCPMC3931228.

17. Shrotriya D, Yadav RS, Srivastava RN. Design and Development of an Indigenous in-house Tissue-Equivalent Female Pelvic Phantom for Radiological Dosimetric Applications. Iranian Journal of Medical Physics. 2018;15:200-5. 
18. International Commission on Radiation Units and Measurements. Dose specification for reporting external beam therapy with photons and electrons. ICRU Report 29. Baltimore: Bethesda; 1978.

19. Akpochafor MO, Madu CB, Habeebu MY, Omo- jola AD, Adeneye S0, Aweda MA. Development of pelvis phantom for verification of treatment planning system using convolution, fast superposition, and superposition algorithms. Journal of Clinical Sciences. 2017;14:74. doi: 10.4103/ jcls.jcls_78_16. 